\title{
Editorial
}

\section{Relation of Sex Steroid Hormones to Genital Tissue Structure and Arousal: A Sampling of Manuscripts Discussing Endocrinologic Sexual Dysfunction}

\author{
International Journal of Impotence Research (2003) 15, 389-390. doi:10.1038/sj.ijir.3901166
}

As I write the last editorial for issue \#15 of the IJIR: The Journal of Sexual Medicine, the International Society for the Study of Women's Sexual Health (ISSWSH), which has a cooperative relationship with the ISSIR, has just concluded its first European conference in Amsterdam. During this 3-day (October 16-19, 2003) multidisciplinary meeting, almost 300 registrants engaged in discussion of over 60 podium and poster presentations on female sexual functions and dysfunctions. In addition, over 20 invited experts were involved in nine state-of-theart lectures and symposia. Many of the expert panelists serve on either the editorial team (Annamaria Giraldi who was the scientific committee chair for ISSWSH), the editorial board (a board meeting was held during the ISSWSH meeting), or as reviewers for the IJIR: The Journal of Sexual Medicine. Many of the presenters agreed to submit manuscripts to the IJIR: The Journal of Sexual Medicine, helping us to continue to broaden our audience in male and female sexual health medicine. The progress and insight into physiology, pathophysiology, diagnosis and treatment in women's sexual health concerns continues exponentially (the next ISSWSH meeting is in Atlanta, GA, October 28-31, 2004) and the IJIR: The Journal of Sexual Medicine is proud to play a frontline role in this new area of sexual medicine.

Among the many take-home messages from the recent ISSWSH meeting in Amsterdam was that sex steroid hormones play an increasingly recognized and critical role in the physiology and pathophysiology of sexual function and dysfunction. Jeffrey Blaustein, PhD, spoke on 'Sex steroid hormone receptors and female sexual behavior' and Kim Wallen, PhD, lectured on 'Androgen modulation of female sexual desire via free estradiol', reinforcing that message. The Second International Consultation on Erectile and Sexual Dysfunctions held in Paris in July 2003 also emphasized the role of sex steroids in male and female central and peripheral sexual function and dysfunction. Researchers have historically investigated the pathophysiological roles of abnormal vascular, neurogenic, anatomic and medication factors in peripheral male and female sexual dysfunction; however, the mechan- istic understanding of how abnormal sex steroid hormones adversely affect peripheral sexual function has lagged behind.

This issue (15.6) contains two lead articles on the topic of sex steroid hormones and peripheral genital tissue structure and function. The article by Shirai et al 'Downregulation of androgen, estrogen and progesterone receptor genes and protein is involved in aging related erectile dysfunction' is the first study to demonstrate, in aging rat crura, that the downregulation of sex hormone receptors, as determined by RT-PCR and immunostaining, is associated with ED, established by recording the mean intracavernosal pressures following nerve stimulation. The authors found that gene and protein expression of sex hormone receptors were significantly lower or absent in the 24-month-old penile crura. The second lead article by Traish et al 'Sex steroid hormones differentially regulate nitric oxide synthase and arginase activities in the proximal and distal rabbit vagina' demonstrated that bilateral oophorectomy altered both tissue nitric oxide synthase (NOS) activity and tissue arginase activity compared to controls in vaginal tissue, and that replacement by androgens and by estrogens reciprocally changed these activities compared to oophorectomy alone or to controls. The authors concluded that, since steroid hormones regulate tissue NOS and arginase activity, vaginal blood flow is dependent, in part, on the hormonal status.

What are the possible mechanisms whereby abnormal hormones adversely influence genital tissue structure and function? Based on vascular and neurologic pathophysiologies, it has been well appreciated that peripheral genital (male and female) dysfunction may be caused by alterations in endothelial function, nerve growth and/or function, smooth muscle growth and/or function, extracellular matrix and fibroblast function. One hypothesis linking endocrine hormonal influences to the genital tissue structure and function is based on new observations that sex steroid hormones are independently responsible for expression of the relevant proteins and growth factors (vascular endothelial growth factor, transforming growth factor beta, connective tissue growth factor, smooth 
muscle growth factor, epithelium growth factor) that maintain the genital tissue structure and function. In other words, maintaining the hormonal milieu, similar to maintaining the vascular and neurologic integrity, is an important consideration for healthy male and female peripheral genital tissue structure and function.

Several articles ${ }^{1-5}$ concerning mechanistic studies in sex steroid hormones and sexual function/ dysfunction have been published recently in the IJIR: The Journal of Sexual Medicine, consistent with the contemporary nature of this physiological/ pathophysiological association. In 2001, Park et $a l^{1}$ reported on three groups of female New Zealand White rabbits (control and bilateral oophorectomy with and without estrogen replacement). Those animals in the oophorectomy group had significantly less genital blood flow and, histologically, had significantly less genital smooth muscle compared to the other groups, leading to the conclusion that estradiol played an important role in female genital structure and function. Also, in 2001, Yoon et $a{ }^{2},{ }^{2}$ in similar groups of female rabbits, measured nitric oxide synthase (NOS) activity and expression of neural NOS and endothelial NOS using biochemical and Western blot analyses. They reported that oophorectomy significantly increased tissue NOS activity as well as eNOS and nNOS expression, concluding that the estrogen hormonal milieu influences female genital tissue structure and function. In 2003, Adaiken et $a l^{3}$ reported on 60 Sprague-Dawley male rats divided into three groups of control, low- and high-dose estradiol-administered animals. They found the high estradiol-treated male rats had prolonged mount, intromission and ejaculation latencies, impaired intracavernosal pressure responses to nerve stimulation and increased connective tissue on histology. The hormonal milieu was related to male genital tissue structure and function.

Also, in 2003, Guay et $a l^{4}$ reported in 178 men with secondary hypogonadism and erectile dysfunction that 4 months of clomiphene citrate administration significantly increased luteinizing hormone (LH) and free testosterone (FT) levels. A total of 75\% of the men who received clomiphene improved the erectile function, with the least increase in sexual function in those men with diabetes mellitus, hypertension, coronary artery disease and those on multiple medications. The hormonal milieu affected the sexual function and the adverse vascular influences limited the hormonal-induced sexual improvement. Finally, a less known but interesting article by Gomaa et $a l^{5}$ in 2001 reported on 42 men with erectile dysfunction and low normal or mildly abnormal testosterone levels, who received either a topical cream with testosterone alone or a topical cream with testosterone and vasodilators. While the testosterone levels increased in both groups, the ability to have increased blood flow as well as full erections and intercourse was significantly higher in those who received both testosterone and vasoactive agent. In the two manuscripts investigating human subjects, the inexorable linking of hormones and vascular responses to peripheral genital function was concluded.

Prior to the next series of issues \#16 in the IJIR: The Journal of Sexual Medicine, it is appropriate to examine where we were, because this helps us view where we may be going. As there are valuable contributions on hormonal influences affecting genital structure and function in the IJIR: The Journal of Sexual Medicine, so too are there numerous manuscripts in other relevant areas of male and female sexual function and dysfunction. I remind you that this is your sexual medicine journal; so read your journal faithfully, contribute manuscripts often, cite it routinely, and contact us whenever you feel necessary.

I Goldstein Editor

\section{References}

1 Park $\mathrm{K}$ et al. Decreased circulating levels of estrogen after vaginal and clitoral blood flow and structure in the rabbit. Int $J$ Impot Res 2001; 13: 116-124.

2 Yoon $\mathrm{HN}$ et al. Effects of estrogen on nitric oxide synthase and histological composition in the rabbit clitoris and vagina. Int $J$ Impot Res 2001; 13: 205-211.

3 Adaikan PG, Srilatha B. Oestrogen-mediated hormonal imbalance precipitates erectile dysfunction. Int J Impot Res 2003; 15: $38-43$.

4 Guay AT, Jacobson J, Perez JB, Hodge MB, Velasquez E. Clomiphene increases free testosterone levels in men with both secondary hypogonadism and erectile dysfunction: who does and does not benefit? Int J Impot Res 2003; 15: 156-165.

5 Gomaa A, Eissa M, El-Gebaley A. The effect of topically applied vasoactive agents and testosterone versus testosterone in the treatment of erectile dysfunction in aged men with low sexual interest. Int J Impot Res 2001; 13: 93-99. 